Chirurg 2017 $88: 729-730$

DOI 10.1007/s00104-017-0475-3

(c) Springer Medizin Verlag GmbH 2017

CrossMark

\section{H. Dralle}

Sektion Endokrine Chirurgie, Klinik für Allgemein-, Viszeral- und Transplantationschirurgie, Universitätsklinikum Essen, Essen, Deutschland

\title{
Die R1-Resektion in der viszeralonkologischen Chirurgie
}

Während die TNM/pTNM-Klassifikation die Tumorausdehnung bezogen auf Primärtumor, Lymphknoten und Fernmetastasen definiert, beschreibt die Residualtumorklassifikation (R-Klassifikation) therapiebezogene Kategorien, unabhängig davon, ob es sich um chirurgische, radio- oder chemotherapeutische Therapien handelte (Pollheimer und Langner). Die Kategorien der R-Klassifikation beurteilen die Effektivität der Therapie, sie können damit Art und Ausmaß weiterer Diagnostik- und/oder Therapiemaßnahmen beeinflussen. Die R-Klassifikation hat nicht zuletzt in Abhängigkeit von der Art des Tumors auch prognostische Bedeutung.

\section{) An der Resektionslinie endet der Chirurg und beginnt der Pathologe}

An der Resektionslinie endet der Chirurg und beginnt der Pathologe. Die von der R-Klassifikation beabsichtigte Therapierelevanz ergibt sich aber nur dann, wenn an der Schnittstelle zwischen beiden eine enge Kooperation und ein Konsens über die Methoden zur Feststellung des R-Status von chirurgischer und pathologischer Seite bestehen. Vor diesem Hintergrund soll das im vorliegenden Heft gewählte Schwerpunktthema eine aktuelle Standortbestimmung vornehmen und für wichtige Organtumoren der Onkologie resümieren, welche Voraussetzungen an die Festlegung der einzelnen Kategorien des R-Status geknüpft sind und welche therapeutischen und prognostischen Konsequenzen sich aus der R1-Situation ergeben.
Die Situation der R1-Resektion beim Schilddrüsenkarzinom ist wie bei anderen parenchymatösen Organen, z. B. Pankreaskarzinom, anders, als bei den in diesem Heft ebenfalls abgehandelten Hohlorganresektionen. Es gibt sowohl eine intrathyreoidale Resektionsebene als auch eine zirkumferenzielle, die bei extrathyroidal invasiven Schilddrüsenkarzinomen die gesamte Invasionsfront außerhalb der Schilddrüse umfasst (Synoracki et al.). Hinsichtlich der extrathyroidalen Invasionsebene ist seit längerem die prognostische Relevanz bei geringer vs. ausgedehnterer Infiltration der perithyreoidalen Strukturen umstritten. Da die neue TNM-Klassifikation aus welchen Gründen auch immer die minimal-invasive extrathyroidale Invasion herausgenommen hat, haben die Autoren einen Klassifikationsvorschlag erarbeitet, der hier erstmals publiziert wird und für den geplant ist, ihn in das TNM-Supplement aufzunehmen, damit die prognostische Bedeutung der extrathyroidalen Invasion weiter geklärt werden kann.

\section{》) Die viszeralonkologische Bedeutung der R1-Situation ist abhängig vom Karzinomtyp und seiner Organzuordnung}

Beim Magenkarzinom ist der distale Resektionsrand weniger intensiv untersucht, aber auch die prognostische Bedeutung des proximalen Resektionsrandes umstritten (Pollheimer und Langner). Die aktuelle Datenlage spricht dafür, dass eine R1-Situation vor allem in frühen Tumorstadien von prognostischer Bedeutung ist und dann, wenn nicht bereits intraoperativ aufgrund des Schnellschnittergebnisses nachreseziert wurde, unter kurativer Intention nachoperiert werden sollte. Dieser datengestützten Leitlinienempfehlung wird allerdings bislang zu selten Rechnung getragen (Ridwelski et al.).

Auch beim Ösophaguskarzinom ist wie $\mathrm{zu}$ erwarten die R1-Situation prognosebestimmend. Bezüglich des CRM („circumferential resection margin“) liegen allerdings im Gegensatz zum oralen und aboralen Resektionsrand keine systematischen Studien vor, auch sind die Definitionen des CRM seitens der englischen und amerikanischen Pathologen unterschiedlich (Gockel und Wittekind). In jedem Fall schafft die spezielle Anatomie des Ösophagus, die technischen Besonderheiten seines Ersatzes und die potenzielle Morbidität des Eingriffs eine Situation, die bei Vorliegen einer R1-Resektion am besten im Tumorboard diskutiert wird.

Die prognostische Bedeutung der R1Situation beim Pankreaskarzinom ist in den letzten Jahren bekanntlich vielfach kontrovers diskutiert worden. Sicher ist, dass R1-Situationen unter Berücksichtigung des CRM wesentlich häufiger sind, als früher bei eindimensionaler Resektionsrandhistologie diagnostiziert wurde. Die standardisierte pathohistologische Bearbeitung der Resektate ist daher von ausschlaggebender Bedeutung (Pollheimer und Langner; Weber et al.). Während in der aktuellen TNM-Klassifikation R1 als mikroskopischer Tumorzellnachweis am CRM definiert wird, hat die aktuelle S3-Leitlinie zum Pankreaskarzi- 
nom die Empfehlung des Royal College of Pathologists übernommen, welche einen minimalen Tumorabstand zum Resektionsrand von $<1 \mathrm{~mm}$ als R0/CRM-positiv zu klassifizieren empfiehlt. Die Indikation zur intraoperativen Nachresektion bei einem R1-Schnellschnitt ist in der Regel gegeben, soweit es sich nicht um individuellen Indikationen vorbehaltene Multiviszeral- oder arterielle Gefäßresektionen handelt. Inwieweit die postoperativpathohistologische Feststellung einer R1Situation Einfluss auf das therapeutische Konzept hat, muss in weiteren Studien geklärt werden. Aufgrund bisheriger Daten kann bei Durchführung einer adjuvanten Therapie von einer Überlebensverlängerung ausgegangen werden (Weber et al.).

Der Beitragvon R. Raabzeigt hinsichtlich des Rektumkarzinoms sehr deutlich, dass die R1-Resektion nicht nur die Lokalrezidivrate, sondern auch das Fernmetastasenrisiko negativ beeinflusst. Die Vermeidung einer R1-Situation hat dabei nicht nur einen Bezug zur schichtberücksichtigenden Technik, auch die neoadjuvante Therapie kann dazu beitragen, das Risiko einer R1-Situation zu vermindern.

Wie beim Rektumkarzinom ergibt sich auch bei den anderen in diesem Heft von Der Chirurg beschriebenen Tumorentitäten des Gastrointestinaltraktes, in Analogie auch bei den besprochenen Drüsenkarzinomen mit ihren nichthohlorganbezogenen anatomischen Besonderheiten, hinsichtlich der Faktizität der R1-Situation ein klinisch-pathologisches Kontinuum, das nicht nur das unterschiedliche Ausmaß des oralen, aboralen, und zirkumferenziellen, sondern des gesamten longitudinalen Resektionsrandes einschließt und auch Tumorzellverschleppungen zu berücksichtigen hat. Die detailgerechte Information des Chirurgen an den Pathologen ist für die Befunderfassung essenziell. Je genauer der Pathologe die R-Situation pathologisch-anatomisch lokalisiert und quantifiziert, desto kleiner ist das für die Prognose und Nachsorge so wichtige
„Delta“ zwischen Befund und dem zu kalkulierenden Rezidivrisiko.

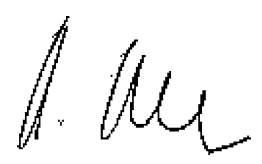

Prof. Dr. med. Dr. h.c. mult. Henning Dralle

\section{Korrespondenzadresse}

Prof. Dr. med. Dr. h.c. mult. H. Dralle, FRCS, FACS, FEBS

Sektion Endokrine Chirurgie, Klinik für Allgemein-, Viszeral- und Transplantationschirurgie, Universitätsklinikum Essen Hufelandstr. 55, 45147 Essen, Deutschland henning.dralle@uk-essen.de

Interessenkonflikt. H. Dralle gibt an, dass kein Interessenkonflikt besteht.
Auch Online-Zugang zu allen Beiträgen Ihres Zeitschriftenabonnements

Wussten Sie, dass Sie als Abonnent dieser Zeitschrift automatisch online Zugriffsrechte auf das gesamte Beitragsarchiv und die CME-Kurse haben?

Der Zugang zu Ihrer Online-Bibliothek und den CME-Kursen ist im Abonnement Ihrer Zeitschrift inbegriffen. Für den Zugang müssen Sie sich lediglich einmal über www.springermedizin.de/register registrieren.

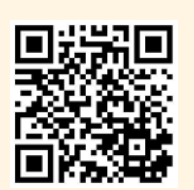

Über diesen QR-Code schnell und einfach registrieren

Bitte nutzen Sie für die Registrierung Vorund Nachname und Lieferadresse wie beim Abonnement der Zeitschrift (s. Adressaufkleber auf Ihrem Heft). So kann im System die Zugehörigkeit zu Ihrer Zeitschrift sichergestellt werden.

Aufgrund des Heilmittelwerbegesetzes dürfen die Inhalte der Website nur medizinischen Fachkreisen zur Verfügung gestellt werden. Bei der Anmeldung bitten wir Sie deshalb, einen Berufsnachweis vorzulegen.

Bei Medizinern mit Mitgliedschaft in der deutschen Ärztekammer reicht die einheitliche Fortbildungsnummer

(EFN). Alternativ schicken Sie eine Bestätigung des Arbeitgebers, Studiennachweis oder andere Zeugnisse ganz unkompliziert an kundenservice@springermedizin.de.

Mit Benutzername und Passwort haben Sie außerdem Zugang zu den freien Inhalten auf den Seiten von:

https://www.springermedizin.de/ https://www.aerztezeitung.de/

Sollten Fragen oder Probleme auftauchen, wenden Sie sich einfach an Ihren Kundenservice:

kundenservice@springermedizin.de 